\title{
Gastrointestinal symptoms in COVID-19
}

\author{
Konrad Lewandowski ${ }^{1}$, Magdalena Kaniewska ${ }^{1}$, Mariusz Rosołowski ${ }^{2,3}$, Grażyna Rydzewska ${ }^{1,4}$ \\ ${ }^{1}$ Department of Internal Medicine and Gastroenterology with Inflammatory Bowel Disease Unit, Central Clinical Hospital \\ of Ministry of Inferior and Administration, Warsaw, Poland \\ ${ }^{2}$ Department of Internal Medicine and Hypertension, Medical University of Bialystok, Bialystok, Poland \\ ${ }^{3}$ Department of Hypertension, Gastroenterology, and Internal Medicine, Medical University of Bialystok Clinical Hospital, \\ Bialystok, Poland \\ ${ }^{4}$ Collegium Medicum, Jan Kochanowski University, Kielce, Poland
}

Gastroenterology Rev 2023; 18 (1): 61-66

DOI: https://doi.org/10.5114/pg.2021.112683

Key words: SARS-CoV-2, COVID-19, gastrointestinal symptoms, gut microbiota.

Address for correspondence: Magdalena Kaniewska, Department of Internal Medicine and Gastroenterology with Inflammatory Bowel Disease Unit, Central Clinical Hospital of Ministry of Inferior and Administration, Warsaw, Poland, e-mail: kaniewscy@o2.pl

\begin{abstract}
SARS-CoV-2 infection manifests mainly by involving the respiratory system. Due to the presence of abdominal symptoms, the digestive system is clearly involved in the expression, transmission, and possible pathogenesis of COVID-19. There are many theories regarding the development of abdominal symptoms, including angiotensin 2 receptor, cytokine storm, and disturbances of the intestinal microbiome. This paper provides an overview of the most important meta-analyses and publications on gastrointestinal symptoms and the gut microbiome in COVID-19.
\end{abstract}

\section{Introduction}

The 2019 coronavirus disease (COVID-19) can be clinically characterized as a pneumonia that often leads to respiratory failure; the gastrointestinal (GI) tract is strongly involved, which translates into the disease's manifestation and possible transmission. Severe acute respiratory syndrome coronavirus 2 (SARS-CoV-2) enters a host cell through a receptor on its surface. This receptor is the angiotensin-converting enzyme 2 receptor, which is more numerous in the Gl tract than in the respiratory system [1-3]. The genetic material of the virus has been detected in stool in up to $50 \%$ of patients. It is possible that an intestinal infection may adversely affect lung infection through changes in the intestinal microbiome, the phenomenon of cytokine storm, and increased intestinal permeability [1, 4-8]. Abdominal symptoms are common among COVID-19 patients, but reports regarding their frequency or impact on disease course are contradictory.

Studies published at the beginning of the pandemic suggested that GI symptoms occur in less than $10 \%$ of patients; this was confirmed in the last 2 meta-analyses [9-13]. However, the results of most studies suggest that abdominal symptoms are more common - from
$30 \%$ to $60 \%$ [14-17]. The issue of abnormal laboratory test results concerning liver function has also been raised, estimated to occur in $15 \%$ to $50 \%$ of cases [ 9 , $12,13,15]$. The effect of $\mathrm{Gl}$ involvement on the severity of COVID-19 is still unclear [18-21].

\section{Gastrointestinal symptoms}

Cheung et al., in their study and meta-analysis of findings from publications, found that $17.6 \%$ of patients with COVID-19 had GI symptoms. Moreover, virus RNA was detected in the stool samples of $48.1 \%$ of patients. Among $25.4 \%$ of patients who presented Gl symptoms, the most common symptom was diarrhoea in $22 \%$, followed by abdominal pain/discomfort in $11.9 \%$, and vomiting in $1.7 \%$ of patients. Stool viral RNA was found in $15.3 \%$ of cases, and it was higher among those with diarrhoea. For the meta-analysis of all Gl symptoms, Cheung et al. took into consideration 4243 patients with COVID-19 from 60 studies. The most common symptom, described by $26.8 \%$ of the cohort, was lack of appetite, which is not very specific. Diarrhoea was the second most common, in $12.5 \%$ of patients, followed by nausea/vomiting in $10.2 \%$, and abdominal pain/discomfort in $9.2 \%$. Significant hetero- 
geneity was seen for anorexia, nausea/vomiting, and diarrhoea. Chueng et al. concluded that the actual prevalence of any Gl symptoms could be underestimated because many earlier studies did not report symptoms other than diarrhoea, and they also pointed to the probability of faecal transmission. They also showed many similarities to SARS and MERS infections [22].

In a multicentre cohort study across 9 hospitals in the United States, a total of 318 patients with confirmed COVID-19 were included. Overall, $61.3 \%$ of the patients reported at least $1 \mathrm{Gl}$ symptom on presentation; the most common were loss of appetite (34.8\%), diarrhoea (33.7\%), and nausea (26.4\%) [16]. The frequency of Gl symptoms in this study was higher than in studies from China.

In the largest meta-analysis, which was performed by Tariq et al., a total of 78 studies with 12,797 patients were included. Among the Gl symptoms, loss of appetite occurred in $22.3 \%$ of the individuals, diarrhoea in $12.4 \%$, nausea/vomiting in $9.0 \%$, and abdominal pain in $6.2 \%$. Mortality in the group of GI symptoms was $0.4 \%$, which was similar to the overall mortality $(2.1 \%$; $p=0.15)$. Significant heterogeneity was reportedin the diarrhoea and nausea/vomiting. They summarized that Gl symptoms are seen up in up to 1 in 5 patients with COVID-19. The highest prevalence was for anorexia, whereas the other symptoms occurred in up to $10 \%$ of patients. Tariq et al. concluded that this must be understood with caution because variable follow-up, lack of uniform criteria for mortality, and lack of adjustment for confounders would prevent proper estimation of mortality. The amount of diarrhoea and nausea/vomiting were lower in the studies from China than in studies conducted outside China, probably due to increasing knowledge and awareness of the presence of GI symptoms. They pointed out that patients with mild disease were not admitted to the hospital, which could affect estimates of symptom prevalence. Also, the exclusion of this group means that Gl symptoms may beunderreported. Moreover, in the included studies there was no information on Gl-specificlaboratory tests, endoscopy reports, histopathology reports, or imaging. The overall mortality was similar to the mortality in patients with GI symptoms, so there was no uniform definition for COVID-19-attributable mortality [23]. Standardization is crucial, so the following should be defined: death due to the infection itself or to comorbid disease, and the time frame. The systemic review did not assess the effect of different factors, such as age or comorbid diseases.

Elmunzer et al. examined the occurrence and influence of abdominal symptoms on the course of COVID-19. 1992 patients from 36 centres were included in the study. A regression analysis was performed to assess the association between digestive symptoms and the severity of COVID-19. Of the 1992 patients, 53\% experienced at least $1 \mathrm{Gl}$ symptom at any time during the disease, the most common ones being diarrhoea (34\%), nausea (27\%), vomiting (16\%), and abdominal pain (11\%). In $74 \%$ of the cases, the Gl symptoms were mild. After adjusting for potential confounders, the presence of Gl symptoms at any time (odds ratio (OR) $=0.93 ; 95 \% \mathrm{Cl}: 0.76-1.15)$ or liver test abnormalities on admission ( $\mathrm{OR}=1.31 ; 95 \% \mathrm{Cl}$ : 0.80-2.12) were not associated independently with mechanical ventilation or death. In their conclusions, they emphasized that GI symptoms and liver function test abnormalities were common, but most were mild and not associated with a more severe clinical course [24].

\section{The concept of the gut-lung axis in COVID-19}

In the human gut, the existing microbiome consists of $10^{14}$ microorganisms, including bacteria, archaea, viruses, and fungi [25]. Bacteria of the genera Actinobacteria, Firmicutes, Proteobacteria, and Bacteroidetes should dominate the composition of the normal intestinal microbiome [26]. It positively influences various physiological functions in the human body and exerts a key role through its protective, trophic, immunomodulatory, and metabolic effects. The presence of beneficial microorganisms in the respiratory tract, mainly in the lungs, has also been described [27]. Bacteroidetes and Firmicutes are considered to be the dominant bacteria in the intestine. A similar relationship has been proven in the respiratory tract where Bacteroidetes, Firmicutes, and Proteobacteria are present [28]. The relationship between the presence of similar strains of bacteria in the intestine and the lungs is associated with the exchange between the intestinal microbiota and the lungs and is defined as the "gut-lung axis" [29]. The fact that endotoxins and metabolites produced by bacteria (e.g. during pneumonia) in the bloodstream can induce changes in the intestinal microbiome suggests that this axis is bidirectional [30]. A deterioration in the composition of the intestinal microbiome has been demonstrated in respiratory infections [31]. The theory that improving the composition of the gut microbiome may benefit and reduce inflammation in the lungs seems to be valid. Current data show that disturbance of the gut microbiome during infection with SARS-CoV-2. COVID-19 is most often pneumonia, which can sometimes lead to acute respiratory distress syndrome (ARDS) [32]. There are reportsof a role of the gut microbiome in the pathogenesis of sepsis and ARDS [33]. Additionally, it is well known that deterioration of the quality of the 
intestinal microbiome, i.e. dysbiosis, may thenbe associated with many diseases or may worsen the course of comorbidities [34].

\section{Gut microbiota in COVID-19}

Zuo et al. investigated changes in the intestinal microbiome of patients with confirmed SARS-CoV-2 infection during hospitalization and assessed the relationship between the disease severity and the excretion of viral genetic material in the faeces. They performed shotgun metagenomic sequencing on faecal samples from 15 COVID-19 patients. During hospitalization, samples were taken 2 or 3 times a week. Patients with SARS-CoV-2 infection had significant changes in their gut microbiome compared with the controls, characterized by an enrichment of opportunistic pathogens and a depletion of beneficial commensals during hospitalization and at all time-points during hospitalization. Depleted symbiotes and gut dysbiosis persisted even after SARS-CoV-2 infection. An abundance of Coprobacillus spp., Clostridium ramosum, and Clostridium hathewayi at baseline correlated with the severity of COVID-19; there is an inverse correlation between the abundance of Faecalibacterium prausnitzii (an anti-inflammatory bacterium) and theseverity of the disease. During hospitalization, Bacteroides dorei, Bacteroides thetaiotaomicron, Bacteroides massiliensis, and Bacteroides ovatus - which downregulate angiotensin-2-converting enzyme expression in the gut of mice - inversely correlated with SARS-CoV-2 burden in patients' stool samples. They found permanent changes in the fecal microbiome during hospitalization compared with the control group. The changes in the microbiota were associated with the levels of SARS-CoV-2 and COVID-19 in the faeces. Moreover, the reduction of ACE2 expression in the intestines of mice showed a significant inverse correlation with the viral load of SARS-CoV-2 in the faeces of COVID-19 patients [35].

\section{Probiotics in COVID-19}

The response to SARS-CoV-2 infection may be regulated by the gut microbiome, which is instrumental in maintaining optimal immune function by preventing an excessive inflammatory response. Microorganisms serve as a source of microorganism-associated molecular patterns (MAMPs) as well as pathogen-associated molecular patterns (PAMPs). Both are recognizable on host cells by pattern recognition receptors (PRRs), which include Toll-like receptors (TLRS) and nucleotide binding receptors [36]. TLRs recognize MAMPS and PAMPs among other molecules and elicit different immune responses depending on the cell type: ligand or receptor. PRR training with expression of innate cells with micro- bial/non-microbial ligands of the intestine is needed as a protective mechanism that is independent of adaptive immunity during secondary infection or pathogen exposure [36]. The metabolites secreted by the gut microbiota and immunomodulatory signals, which include short chain fatty acids - such as butyrate, acetate, and propionate - and secondary bile acids secreted by commensals such as Bifidobacteria spp., bind to their receptors in cells and macrophages, thereby modulating their metabolism and functions [37]. The introduction of probiotic strains such as Bifidobacterium lactis into healthy elderly volunteers resulted in a significant increase in the percentage of mononuclear leukocytes and the anti-tumour activity of NK cells [38]. The composition of a balanced gut microbiota has a great influence on the effectiveness of lung immunity [27]. Disturbances in the gut microbiome due to the widespread use of antibiotics may alsohave a similar effect as that observed in population studies where the increased use of penicillins, cephalosporins, macrolides, and quinolones correlated with an increased risk of lung cancer in humans [39]. Probiotics have shown good results in improving inflammation as well as regulating innate immunity via TLRs and the relevant signalling pathways [40]. Studies based on a mouse model have shown that Treg cells, which downregulate allergic response, can beinduced by the administration of probiotic bacteria such as Lactobacillus rhamnosus, Bifidobacterium lactis, and Bifidobacterium breve [41]. One conclusion suggests that modulation of the gut microbiota may affect immunity during SARS-CoV-2 infection.

\section{ACE-2 receptor in COVID-19}

The ACE2 receptor, through which the virus enters a cell, has many functions in the GI tract, regulating intestinal amino acid homeostasis, modulating the intestinal microbiome, and influencing the expression of antimicrobial peptides, among other things [42]. It has been found that COVID-19 running may reduce the amount of ACE2 in various tissues, which, as with functional diseases, may cause irritable bowel syndrome, disorders of the gut microbiome, and micro-inflammation in the digestive tract, for example [43]. This theory largely explains the occurrence of ailments such as abdominal pain and diarrhoea. In addition, blocking ACE2 appears to be a potential treatment option for SARSCoV-2, and research is currently underway. Among the new compounds under development is human recombinant soluble ACE-2 (hrs-ACE-2 [APN01; Apeiron Biologics, Vienna, Austria]), which has 2 mechanisms of action that should theoretically be beneficial for COVID-19. The first is to bind the viral peak protein and thus neutralize SARS-CoV-2, and the second is to minimize dam- 
age to many organs, including the lungs, kidneys, and heart, due to the unrelenting hyperactivation of the renin-angiotensin system and increased angiotensin II levels. ACE-2 administration for 4-6 h was tested in 89 patients, namely, healthy volunteers in phase I studies and patients with ARDS in phase II clinical studies, with an acceptable safety profile. Moreover, hrs-ACE-2 can reduce the SARS-CoV-2 load 1000 to 5000 times in in vitro cell culture and modified organoid experiments, which directly shows that ACE2 can effectively neutralize SARS-CoV-2 [44].

\section{Cytokine storm in COVID-19}

The term cytokine release syndrome (CRS), more commonly known as cytokine storm, is used to describe the abnormal secretion of mediators and associated immunopathological events that occur after severe bacterial and viral infections. CRS is associated with an excessive pro-inflammatory response and an ineffective anti-inflammatory control mechanism, leading to tissue damage. The human immune system plays a pivotal role in eliminating infectious agents such as influenza and coronaviruses by recruiting leukocytes and releasing cytokines. The limited and well-harmonized stimulation of immune responses is usually the body's first mechanism of action to build up defence against any viral infection. Nevertheless, an unregulated and over-exaggerated immune response can alter immune function, leading to tissue damage and organ failure. Gu et al. postulated that the production of various cytokines that cause cytokine storms in SARS-CoV-2 patients causes immunopathogenic injuries [45]. Therefore, effectively lowering the levels of pro-inflammatory cytokines in patients with severe COVID-19 is crucial in order to prevent the deterioration of their health. Hojyo et al. confirmed that during SARS-CoV-2 infection, the alveolar epithelial cells, macrophages, and circulating monocytes are activated by TLRs, which are among the larger set of PRRs. Thanks to their immune memory, they recognize the threat and then produce a large number of inflammatory cytokines and chemokines that attract even more immune cells, especially monocytes and T cells, causing widespread pneumonia [46]. Xu et al. presented the results of autopsies in COVID-19 patients, in which lung-dominated interstitial mononuclear inflammatory infiltrates and severe lymphopaenia with hyperactivated T cells in the peripheral blood were found [47]. Moreover, Qin et al. demonstrated that COVID-19 patients also had a lower level of regulatory $T$ lymphocytes, which were more clearly lowered in cases of severe infections [48]. Huang et al. proved that patients hospitalized due to a severe course of COVID-19 showed high levels of IL-2, IL-7, IL-10, IL-18, G-CSF, TNF, CXCL10, MCP1, and MIP1 $\alpha$ in the serum, which confirms that COVID-19 may be associated with cytokine storming [10].

These findings have led to the hypothesis that the main cause of death in COVID-19 is ARDS due to the cytokine storm. Intravascular coagulation was one of the causes of multi-organ damage mediated mainly by inflammatory cytokines, in particular IL-6 [10, 47, 48]. Patients presented multi-organ failure with coagulation disorders represented by lower platelet counts and increased levels of D-dimers, which were associated with poor prognosis and could explain the presence of microclots in the lungs, limbs, brain, heart, Gl organs, and kidneys. Park et al. recognized cell apoptosis induced by SARS-CoV-2 infection as another cause of multi-organ failure, leading to vascular leakage and inducing a cytopathic effect on cells [49]. It therefore appears that disease worsening or mortality may also be due to a cytokine storm, including ARDS, from viral lung infection, which is responsible for whole-body multi-organ failure. These inflammatory mediators can also lead to vascular hyperpermeability and may stimulate ACE2-expressing endothelial cells in arteries and veins, which together with viral particles cause systemic inflammation.

\section{Conclusions}

We need to understand every possible pathomechanism in the course of COVID-19 as soon as possible. The presence of SARS-CoV-2 RNA in the stool in some patients, as well as Gl symptoms and disturbances in the intestinal microbiome, may play an important role in determining the course of this disease. Medicines used to treat COVID-19 can also be considered a cause of Gl symptoms, because azithromycin, lopinavir, and ritonavir often cause side effects of diarrhoea, abdominal pain, or nausea [50]. Elderly people, immunocompromised patients, and patients with other comorbidities such as type 2 diabetes mellitus or cardiovascular disorders have a much more severe disease course and a significantly higher risk of death.

Studies published at the beginning of the pandemic suggested that GI symptoms occur in less than $10 \%$ of patients [9-13]. However, the results of most studies suggest that abdominal symptoms are more common, from $30 \%$ to $60 \%$ [14-17]. The issue of abnormal liver function laboratory test results has also been raised, which is estimated to occur in $15 \%$ to $50 \%$ of cases [ 9 , $12,13,15]$. The effect of Gl involvement on the severity of COVID-19 is still unclear [18-21]. Most studies to date have reported that the presence of abdominal symptoms worsens the course of COVID-19. The reports by Livanos et al. and the extensive work carried out in North American centres by Elmunzer et al. seem to be an exception. Livanos et al. postulated a theory based 
on the protective role of $\mathrm{Gl}$ symptoms on the course of COVID-19. The presence of these symptoms attenuates SARS-CoV-2 and leads to a lower inflammatory response, which in turn reduces the clinical course of the disease [51]. Elmunzer et al. concluded that GI symptoms and liver test abnormalities were common in patients hospitalized with COVID-19, but that the majority were mild and not associated with a more severe clinical course [25]. The current research, however, requires validation; meta-analyses are necessary to clearly establish how abdominal symptoms affect the prognosis of COVID-19 patients.

It is worth noting that in patients with COVID-19, there are numerous data on disturbances in the gut microbiome, so there is a possibility that intestinal dysbiosis may also influence the clinical symptoms of COVID-19. Many probiotics have been shown to improve or relieve lung disease by modulating the immune system. The best way to modulate it is by following a balanced diet, which should be refined depending on the patient's needs. This may improve and accelerate the recovery of patients, especially elderly or immunocompromised people infected with SARS-CoV-2. An effective nutritional strategy and specific functional food targeting the gut microbiome for a specific population group can significantly improve the prognosis of COVID-19 patients. It certainly requires more careful research that can help us in the unequal fight against SARS-CoV-2.

\section{Conflict of interest}

The authors declare no conflict of interest.

\section{References}

1. Xiao F, Tang M, Zheng X, et al. Evidence for gastrointestinal infection of SARS-CoV-2. Gastroenterology 2020; 158: 1831-3.

2. Du M, Cai G, Chen F, et al. Multiomics evaluation of gastrointestinal and other clinical characteristics of SARS-CoV-2 and COVID-19. Gastroenterology 2020; 158: 2298-3230.

3. ACE2 angiotensin I converting enzyme 2 [Homo sapiens (human)]. Gene ID: 59272. Available from: https://www.ncbi.nlm. nih.gov/gene/59272. Accessed May 9, 2020.

4. Han C, Duan C, Zhang S, et al. Digestive symptoms in COVID19 patients with mild disease severity: clinical presentation, stool viral RNA testing, and outcomes. Am J Gastroenterol 2020; 115: 916-23.

5. Wu Y, Guo C, Tang L, et al. Prolonged presence of SARS-CoV-2 viral RNA in faecal samples. Lancet Gastroenterol Hepatol 2020; 5: 434-5.

6. Bradley KC, Finsterbusch K, Schnepf D, et al. Microbiota driven tonic interferon signals in lung stromal cells protect from influenza virus infection. Cell Rep 2019; 28: 245-56.

7. Marsland BJ, Trompette A, Gollwitzer ES. The gut-lung axis in respiratory disease. Ann Am Thorac Soc 2015; 12 (Suppl 2): s150-6.
8. Mönkemüller K, Fry L, Rickes S. COVID-19, coronavirus, SARSCoV-2 and the small bowel. Rev Esp Enferm Dig 2020; 112 : 383-8.

9. Guan WJ, Ni ZY, Hu Y, et al. Clinical characteristics of coronavirus disease 2019 in China. N Engl J Med 2020; 382: 1708-20.

10. Huang C, Wang Y, Li X, et al. Clinical features of patients infected with 2019 novel coronavirus in Wuhan, China. Lancet 2020; 395: 497-506.

11. Jin X, Lian JS, Hu JH, et al. Epidemiological, clinical and virological characteristics of 74 cases of coronavirus-infected disease 2019 (COVID-19) with gastrointestinal symptoms. Gut 2020; 69: 1002-9

12. Sultan S, Altayar O, Siddique S, et al. AGA Institute rapid review of the GI and liver manifestations of COVID-19, meta-analysis of international data, and recommendations for the consultative management of patients with COVID-19. Gastroenterology 2020; 159: 320-34.e27.

13. Parasa S, Desai M, Thoguluva Chandrasekar V, et al. Prevalence of gastrointestinal symptoms and fecal viral shedding in patients with coronavirus disease 2019: a systematic review and meta-analysis. JAMA Netw Open 2020; 3: e2011335.

14. D’Amico F, Baumgart DC, Danese S, et al. Diarrhea during COVID-19 infection: pathogenesis, epidemiology, prevention and management. Clin Gastroenterol Hepatol 2020; 18: 1663-72.

15. Aroniadis OC, DiMaio CJ, Dixon RE, et al. Current knowledge and research priorities in the digestive manifestations of COVID-19. Clin Gastroenterol Hepatol 2020; 18: 1682-4.

16. Redd WD, Zhou JC, Hathorn KE, et al. Prevalence and characteristics of gastrointestinal symptoms in patients with SARSCoV-2 infection in the United States: a multicenter cohort study. Gastroenterology 2020; 159: 765-7.e2.

17. Hajifathalian K, Krisko T, Mehta A, et al. Gastrointestinal and hepatic manifestations of 2019 novel coronavirus disease in a large cohort of infected patients from New York: clinical implications. Gastroenterology 2020; 159: 1137-40.e2.

18. Zhang C, Shi L, Wang FS. Liver injury in COVID-19: management and challenges. Lancet Gastroenterol Hepatol 2020; 5 : 428-30.

19. Pan L, Yang P, Sun Y, et al. Clinical characteristics of COVID-19 patients with digestive symptoms in Hubei, China: a descriptive, cross-sectional, multicenter study. Am J Gastroenterol 2020; 115: 766-73.

20. Wei XS, Wang X, Niu YR, et al. Diarrhea is associated with prolonged symptoms and viral carriage in COVID-19. Clin Gastroenterol Hepatol 2020; 18: 1753-9.e2.

21. Mao R, Qiu Y, He JS. Manifestations and prognosis of gastrointestinal and liver involvement in patients with COVID-19: a systematic review and meta-analysis. Lancet Gastroenterol Hepatol 2020; 5: 667-78.

22. Cheung KS, Hung IFN, Chan PPY, et al. Gastrointestinal manifestations of SARS-CoV-2 infection and virus load in fecal samples from a Hong Kong Cohort: systematic review and meta-analysis. Gastroenterology 2020; 159: 81-95.

23. Tariq R, Saha S, Furqan F, et al. Prevalence and mortality of COVID-19 patients with gastrointestinal symptoms: a systematic review and meta-analysis. Mayo Clin Proc 2020; 95 : $1632-48$. 
24. Elmunzer BJ, Spitzer RL, Foster LD, et al. Digestive manifestations in patients hospitalized with coronavirus disease 2019 Clin Gastroenterol Hepatol 2021; 19: 1355-65.

25. Gill SR. Metagenomic analysis of the human distal gut microbiome. Science 2006; 312: 1355-59.

26. Villanueva-Millán MJ, Pérez-Matute P, Oteo JA. Gut microbiota: a key player in health and disease. A review focused on obesity. J Physiol Biochem 2015; 71: 509-25.

27. Bingula R. Desired turbulence? Gut-lung axis, immunity, and lung cancer. J Oncol 2017; 2017: 5035371.

28. Zhang D. The cross-talk between gut microbiota and lungs in common lung diseases. Front Microbiol 2020; 11: 301.

29. Keely S, Talley NJ, Hansbro PM. Pulmonary-intestinal cross-talk in mucosal inflammatory disease. Mucosal Immunol 2012; 5 : 7-18.

30. Dumas A. The role of the lung microbiota and the gut-lung axis in respiratory infectious diseases. Cell Microbiol 2018; 20: e12966.

31. Groves HT. Respiratory viral infection alters the gut microbiota by inducing inappetence. mBio 2020; 11: e03236-19.

32. Lake MA. What we know so far: COVID-19 current clinical knowledge and research. Clin Med 2020; 20: 124-7.

33. Dickson RP, Arbor A. The microbiome and critical illness. Lancet Respir Med 2017; 4: 59-72.

34. Mosca A, Leclerc M, Hugot JP. Gut microbiota diversity and human diseases: should we reintroduce key predators in our ecosystem? Front Microbiol 2016; 7: 455.

35. Nagpal R. Gut microbiome and aging: physiological and mechanistic insights. Nutr Healthy Aging 2018; 4: 267-85.

36. Ivanov II, Honda K. Intestinal commensal microbes as immune modulators. Cell Host Microbe 2012; 12: 496-508.

37. Rooks MG, Garrett WS. Gut microbiota, metabolites and host immunity. Nat Rev Immunol 2016; 16: 341-52.

38. Gill HS. Enhancement of immunity in the elderly by dietary supplementation with the probiotic Bifidobacterium lactis HN019. Am J Clin Nutr 2001; 74: 833-9.

39. Fagundes CT. Transient TLR activation restores inflammatory response and ability to control pulmonary bacterial infection in Germfree mice. J Immunol 2012; 188: 1411-20.

40. West CE. Bugging allergy; role of pre-, pro- and synbiotics in allergy prevention. Allergol Int 2017; 66: 529-38.

41. Feleszko W. Probiotic-induced suppression of allergic sensitization and airway inflammation is associated with an increase of $T$ regulatory-dependent mechanisms in a murine model of asthma. Clin Exp Allergy 2007; 37: 498-505.

42. Hashimoto T, Perlot T, Rehman A, et al. ACE2 links amino acid malnutrition to microbial ecology and intestinal inflammation. Nature 2012; 487: 477-81.

43. Akiho $\mathrm{H}$, Ihara $\mathrm{E}$, Nakamura K. Low-grade inflammation plays a pivotal role in gastrointestinal dysfunction in irritable bowel syndrome. World J Gastroinest Pathophysiol 2010; 1: 97-105.

44. Zoufaly A, Poglitsch $M$, Aberle $\mathrm{JH}$, et al. Human recombinant soluble ACE2 in severe COVID-19. Lancet Respir Med 2020; 8: 1154-8.

45. Gu T, Zhao S, Jin G, et al. Cytokine signature induced by SARSCoV-2 spike protein in a mouse model. Front Immunol 2021; 11: 621441 .
46. Hojyo S, Uchida M, Tanaka K, et al. How COVID-19 induces cytokine storm with high mortality. Inflamm Regen 2020; 40: 37.

47. Xu Z, Shi L, Wang Y, et al. Pathological findings of COVID-19 associated with acute respiratory distress syndrome [published correction appears in Lancet Respir Med. 2020 Feb 25]. Lancet Respir Med 2020; 8: 420-2.

48. Qin C, Zhou L, Hu Z, et al. Dysregulation of immune response in patients with coronavirus 2019 (COVID-19) in Wuhan, China. Clin Infect Dis 2020; 71: 762-8.

49. Park WB, Kwon NJ, Choi SJ, et al. Virus isolation from the first patient with SARS-CoV-2 in Korea. J Korean Med Sci 2020; 35: e84.

50. Eder P, Łodyga M, Dobrowolska A, et al. Addressing multiple gastroenterological aspects of coronavirus disease 2019. Pol Arch Intern Med 2020; 130: 420-30.

51. Livanos AE, Jha D, Cossarini F, et al. Gastrointestinal involvement attenuates COVID-19 severity and mortality. medRxiv 2020; 2020.09.07.20187666.

Received: 18.12 .2021

Accepted: 27.12 .2021 